\title{
The Three Postulates of the Theory of Everything
}

\author{
Ding-Yu Chung \\ Utica, Michigan, USA \\ Email: dy_chung@yahoo.com
}

Received 18 February 2016; accepted 25 April 2016; published 28 April 2016

Copyright (C) 2016 by author and Scientific Research Publishing Inc.

This work is licensed under the Creative Commons Attribution International License (CC BY). http://creativecommons.org/licenses/by/4.0/

(c) (i) Open Access

\section{Abstract}

The three postulates of the posited dynamic and reversible theory of everything are: 1) the oscillating M-theory postulate for the oscillating matter structure, 2) the digital transitional Higgs-reversed Higgs fields postulate for the digital space structure, and 3) the reversible multiverse postulate for all physical laws and phenomena. The posited theory of everything based on the three postulates explains cosmology, the composition (baryonic matter, dark matter, and dark energy) in the universe, the periodic table of elementary particles (quarks, leptons, and bosons), the galaxy evolution, superconductivity, black hole, thermodynamic, and quantum mechanics. Oscillating M-theory is derived from oscillating membrane-string-particle whose space-time dimension number oscillates between 11D and 10D and between 10D and 4D. Space-time dimension number between 10 and 4 decreases with decreasing speed of light, decreasing vacuum energy, and increasing rest mass. The digital transitional Higgs-reversed Higgs fields are derived from digital attachment-detachment spaces which couple to particles. Under spontaneous symmetry breaking, the coupling of massless particle to zero-energy attachment space (the space for mass) produces the transitional nonzero-energy Higgs field-particle composite which under spontaneous symmetry restoring produces massive particle on zero-energy attachment space with the longitudinal component. The opposite of attachment space is detachment space as the space for kinetic energy and the nonzero-energy reverse Higgs field. The combination of $n$ units of attachment space (denoted as 1) and $n$ units of detachment space (denoted as 0 ) brings about the three digital structures: binary partition space $(1)_{n}(0)_{n}$, miscible space $(1+0)_{n}$, and binary lattice space $(10)_{n}$ to account for quantum mechanics, special relativity, and the force fields, respectively. In the third postulate, all physical laws and phenomena are permanently reversible in the multiverse, and temporary irreversible entropy increase is allowed. Our universe is an asymmetrical dual positive-energy-negative-energy universe where the positive-energy universe on attachment space absorbed the interuniversal void on detachment space to result in the combination of attachment space and detachment space, while the negative-energy universe did not absorb the interuniversal void, resulting in temporary irreversible entropy increase through reversibility breaking, symmetry violation, and low entropy beginning. Guided by the reversible negative-energy universe, 
our dual universe is a globally reversible cyclic dual universe.

\section{Keywords}

The Theory of Everything, M-Theory, Higgs Field, Reverse Higgs Field, Multiverse, Cosmology, Matter Structure, Space Structure, Entropy, Thermodynamic, Cyclic Universe, Interuniversal Void

\section{Introduction}

In this paper, the posited theory of everything involves the dynamic and reversible process of continuingly changing matters and spaces in the reversible multiverse. There are three postulates of the posited dynamic and reversible theory of everything. The first postulate is the oscillating M-theory postulate for the oscillating matter structure. In conventional M-theory, space-time dimensional number (D) is fixed. As a result, the observed 4D results from the compactization of the extra space dimensions in 11D M-theory. However, there is no experimental proof for compactized extra space dimensions, and there are numerous ways for the compactization of the extra space dimensions [1]. In the first postulate, oscillating M-theory is derived from oscillating membrane-string-particle whose space-time dimension number oscillates between 11D and 10D and between 10D and 4D. There are no extra space dimensions and compactization. Space-time dimension number between 10 and 4 decreases with decreasing speed of light, decreasing vacuum energy, and increasing rest mass. 4D has zero vacuum energy. The theoretical calculated masses based on the oscillating M-theory postulate for all elementary particles including quarks, leptons, gauge bosons, the Higgs boson, and the knees-ankles-toe in cosmic rays are in good agreements with the observed values [2]-[4]. Different universes at different times in the multiverse have different space-time dimension numbers.

The second postulate of the theory of everything is the digital transitional Higgs-reversed Higgs fields postulate for the digital space structure. In the convention model, the Higgs field is a nonzero-energy field that is hypothesized to exist permanently in the universe. The problem with such nonzero-energy field is the cosmological constant problem from the huge gravitational effect by the nonzero-energy Higgs field [5]. In the conventional model, the nonzero-energy Higgs field is derived from zero-energy ground state space under spontaneous symmetry breaking. In this paper, to avoid the cosmological problem from the huge gravitational effect by the nonzero-energy Higgs field is to make the Higgs field a transitional field which exists momentarily and to make zero-energy ground state space a permanent zero-energy ground state space. In the second postulate for the digital space structure, such zero-energy ground state space is zero-energy "attachment space" which attaches particles to account for the longitudinal component, mass, and reversible movement. Unlike the conventional model, attachment space actively couples to massless particle. Under spontaneous symmetry breaking, the coupling of massless particle to zero-energy attachment space produces the transitional nonzero-energy Higgs field-particle composite which under spontaneous symmetry restoring produces massive particle on zero-energy attachment space with the longitudinal component. The opposite of attachment space is zero-energy detachment space which detaches particles to account for irreversible kinetic energy. Under spontaneous symmetry breaking, the coupling of massive particle to zero-energy detachment space produces the transitional nonzero-energy reverse Higgs field-particle composite which under spontaneous symmetry restoring produces massless particle on zero-energy detachment space without the longitudinal component. The combination of $n$ units of attachment space (denoted as 1 ) and $\mathrm{n}$ units of detachment space brings about the three digital structures: binary partition

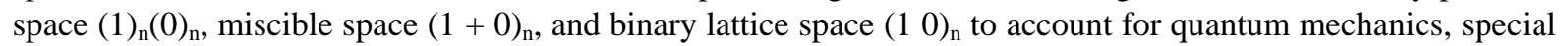
relativity, and the force fields, respectively [6] [7]. Different universes at different times in the multiverse have different spaces. In the second postulate, the digital transitional Higgs-reversed Higgs fields are digital attachment-detachment spaces which couple to particles.

The third postulate of the theory of everything is the reversible multiverse postulate for all physical laws and phenomena. In the second law of thermodynamics, the entropy (a measure of the disorder of a system) of an isolated system can increase, but not decrease. In other words, the entropy of a closed system will never decrease into the future. There are two mysteries about this irreversible entropy increase as described in "From Eternity to Here: The Quest for the Ultimate Theory of Time” by Sean Carroll [8]. Firstly, this irreversible en- 
tropy increase of a macroscopic collection of particles is different from all microscopic reversible processes where for every allowed process there exists a time-reversed process that is also allowed. Secondly, the universe started with the very low entropy state as the inflation-Big Bang in a very small space, not with the high entropy state near equilibrium state in a large space. Such low entropy beginning is a mystery. In this paper, the mysteries of the irreversible entropy increase are explained by the third postulate, the reversible multiverse postulate. In the reversible multiverse postulate, all physical laws and phenomena are permanently reversible, and temporary irreversibility of entropy increase is allowed through reversibility breaking, symmetry violation, and low entropy beginning [7] [9] [10]. The temporary irreversible entropy increase is shown in our universe. Our universe is the dual asymmetrical positive-energy-negative-energy universe where the positive-energy universe on attachment space absorbed the interuniversal void on detachment space to result in the combination of attachment space and detachment space, and the negative-energy universe did not absorb the interuniversal void. Irreversible kinetic energy from detachment space is the source of irreversible entropy increase, so the positiveenergy universe is irreversible, while the negative-energy universe without irreversible kinetic energy from detachment space is reversible. As a result, our whole dual universe is reversible.

The posited theory of everything based on the three postulates explains: 1) cosmology [7] [9]-[11], 2) the composition (baryonic matter, dark matter, and dark energy) in the universe [9] [11] [12], 3) the periodic table of elementary particles [2]-[4] for all elementary particles (quarks, leptons, gauge bosons, the Higgs boson, and the knees-ankles-toe in cosmic rays), 4) the galaxy evolution [13] [14], 5) superconductivity [15], and 6) black hole [16] [17], 7) thermodynamics [7], and 8) quantum mechanics [6] [7]. The theoretical results are in good agreement with the observations, and the calculated values from cosmology, the composition in the universe, and the periodic table of elementary particles are in good agreement with the observed values.

The purpose of this paper is to describe all the three postulates of the theory of everything. Section 2 describes the oscillating reversible M-theory postulate for the oscillating matter structure. Section 3 explains the digital transitional Higgs-reverse Higgs fields postulate for the digital space structure. Cosmology and the reversible multiverse postulate for all physical laws and phenomena are explained in Section 4.

\section{The Oscillating M-Theory Postulate for the Oscillating Matter Structure}

In this paper, the posited theory of everything is the dynamic and reversible process of continuingly changing matters and spaces in the reversible multiverse. Instead of remaining fixed, space-time dimension numbers of matters oscillate. Instead of being passive, spaces actively couple to particles. There are two different spaces whose digital combinations are different under different conditions. In the midst of continuously changing matters and spaces, the multiverse is simple and neat, because the multiverse is permanently reversible to exclude all irreversible physical laws and phenomena. However, temporary irreversible entropy increase is allowed through reversibility breaking, symmetry violation, and low entropy beginning. We live in the universe with such temporary irreversible entropy increase.

The first postulate of the theory of everything is the oscillating M-theory for the oscillating matter structure. M-theory with eleven-dimensional membrane is an extension of string theory with ten-dimensional string, in contrast to the observed 4D. In conventional M-theory with fixed space-time dimension number, the explanation of the hidden extra space dimensions is the compactization of the extra space dimensions, so space-time appears to be 4D. In the first postulate, oscillating M-theory is derived from oscillating membrane-string-particle whose space-time dimension number oscillates between 11D and 10D and between 10D and 4D dimension by dimension reversibly. There is no compactization. Matters in oscillating M-theory include 11D membrane $\left(2_{11}\right)$ as membrane (denoted as 2 for 2 space dimensions) in 11D, 10D string $\left(1_{10}\right)$ as string (denoted as 1 for 1 space dimension) in $10 \mathrm{D}$, and variable $\mathrm{D}$ particle $\left(0_{4}\right.$ to 11$)$ as particle (denoted as 0 for 0 space dimension) in $4 \mathrm{D}$ to $11 \mathrm{D}$.

As described previously [7], the QVSL (quantum varying speed of light) transformation transforms both space-time dimension number (D) and mass dimension number (d). In the QVSL transformation, the decrease in the speed of light leads to the decrease in space-time dimension number and the increase of mass in terms of increasing mass dimension number from 4 to 10 ,

$$
\begin{aligned}
& c_{\mathrm{D}}=c / \alpha^{\mathrm{D}-4}, \\
& E=M_{0} \cdot\left(c^{2} / \alpha^{2(\mathrm{D}-4)}\right)
\end{aligned}
$$




$$
\begin{aligned}
& =\left(M_{0} / \alpha^{2(\mathrm{~d}-4)}\right) \cdot c^{2} . \\
& c_{\mathrm{D}}=c_{\mathrm{D}-n} / \alpha^{2 n}, \\
& M_{0, \mathrm{D}, \mathrm{d}}=M_{0, \mathrm{D}-n, \mathrm{~d}+n} \alpha^{2 n}, \\
& \mathrm{D}, \mathrm{d} \stackrel{\mathrm{QVSL}}{\longrightarrow}(\mathrm{D} \mp n),(\mathrm{d} \pm n) \\
& E_{\text {vacuum }, \mathrm{D}}=E-M_{0, \mathrm{D}} c^{2},
\end{aligned}
$$

where $c_{\mathrm{D}}$ is the quantized varying speed of light in space-time dimension number, $\mathrm{D}$, from 4 to $10, c$ is the observed speed of light in the $4 \mathrm{D}$ space-time, $\alpha$ is the fine structure constant for electromagnetism, $E$ is energy, $M_{0}$ is rest mass, $\mathrm{D}$ is the space-time dimension number from 4 to $10, \mathrm{~d}$ is the mass dimension number from 4 to 10 , $\mathrm{n}$ is an integer, and $E_{\text {vacuum }}=$ vacuum energy. For example, in the QVSL transformation, a particle with 10D4d is transformed to a particle with 4D10d from Equation (1f). Calculated from Equation (1e), the rest mass of 4D10d is $1 / \alpha^{12} \approx 137^{12}$ times of the mass of $10 \mathrm{D} 4 \mathrm{~d}$. In terms of rest mass, $10 \mathrm{D}$ space-time has $4 \mathrm{~d}$ with the lowest rest mass, and 4D space-time has 10d with the highest rest mass. Rest mass decreases with increasing space-time dimension number. The decrease in rest mass means the increase in vacuum energy $\left(E_{\text {vacuum,D }}\right)$, so vacuum energy increases with increasing space-time dimension number. The vacuum energy of $4 \mathrm{D}$ particle is zero from Equation (1g). 11D membrane and 10D string are equal in the speed of light, rest mass, and vacuum energy. Since the speed of light for $>4 \mathrm{D}$ particle is greater than the speed of light for $4 \mathrm{D}$ particle, the observation of $>4 \mathrm{D}$ superluminal particles by 4D particles violates casualty. Thus, $>4 \mathrm{D}$ particles are hidden particles with respect to 4D particles. Particles with different space-time dimensions are transparent and oblivious to one another, and separate from one another if possible.

As described previously [7], in oscillating M-theory, there are two different reversible oscillations: the oscillation between 11D and 10D and the oscillation between 10D and 4D. The reversible oscillation between 11D membrane and 10D string is described in Section 4.The transformation during the oscillation between 10D particle and 4D involves the stepwise two-step transformation: the QVSL transformation and the varying supersymmetry transformation from 10D4d to 4D4d. The QVSL transformation involves the transformation of space-time dimension, $\mathrm{D}$ whose mass increases with decreasing $\mathrm{D}$ for the decrease in vacuum energy. The varying supersymmetry transformation involves the transformation of the mass dimension number, $\mathrm{d}$ whose mass decreases with decreasing $\mathrm{d}$ for the fractionalization of particle, as follows.

stepwise two-step varying transformation

$$
\begin{aligned}
& \text { (1) D,d } \stackrel{\text { QVSL }}{\longrightarrow}(\mathrm{D} \mp 1),(\mathrm{d} \pm 1) \\
& \text { (2) } \mathrm{D}, \mathrm{d} \stackrel{\text { varying supersymmetry }}{\longleftrightarrow} \mathrm{D},(\mathrm{d} \pm 1)
\end{aligned}
$$

The repetitive stepwise two-step transformation between 10D4d and 4D4d as follows.

$$
\text { 10D4d } \leftrightarrow \text { 9D5d } \leftrightarrow \text { 9D4d } \leftrightarrow \text { 8D5d } \leftrightarrow \cdots \leftrightarrow 4 \text { D5d } \leftrightarrow 4 \text { D4d }
$$

In this two-step transformation, the transformation from 10D4d to 9D5d involves the QVSL transformation as in Equation (1d). Calculated from Equation (1e), the mass of 9D5d is $1 / \alpha^{2} \approx 137^{2}$ times of the mass of 10D4d. The transformation of 9D5d to 9D4d involves the varying supersymmetry transformation. In the normal supersymmetry transformation, the repeated application of the fermion-boson transformation carries over a boson (or fermion) from one point to the same boson (or fermion) at another point at the same mass. In the "varying supersymmetry transformation", the repeated application of the fermion-boson transformation carries over a boson from one point to the boson at another point at different mass dimension number in the same space-time number. The repeated varying supersymmetry transformation carries over a boson $B_{d}$ into a fermion $F_{d}$ and a fermion $F_{d}$ to a boson $B_{d-1}$, which can be expressed as follows

$$
\begin{gathered}
M_{\mathrm{d}, \mathrm{F}}=M_{\mathrm{d}, \mathrm{B}} \alpha_{\mathrm{d}, \mathrm{B}}, \\
M_{\mathrm{d}-1, \mathrm{~B}}=M_{\mathrm{d}, \mathrm{F}} \alpha_{\mathrm{d}, \mathrm{F}},
\end{gathered}
$$

where $M_{\mathrm{d}, \mathrm{B}}$ and $M_{\mathrm{d}, \mathrm{F}}$ are the masses for a boson and a fermion, respectively, $\mathrm{d}$ is the mass dimension number, and $\alpha_{\mathrm{d}, \mathrm{B}}$ or $\alpha_{\mathrm{d}, \mathrm{F}}$ is the fine structure constant that is the ratio between the masses of a boson and its fermionic 
partner. Assuming $\alpha$ 's are the same, it can be expressed as

$$
M_{\mathrm{d}, \mathrm{B}}=M_{\mathrm{d}+1, \mathrm{~B}} \alpha_{\mathrm{d}+1}^{2}
$$

The mass of 9D4d is $\alpha^{2} \approx(1 / 137)^{2}$ times of the mass of 9D5d through the varying supersymmetry transformation. The transformation from a higher mass dimensional particle to the adjacent lower mass dimensional particle is the fractionalization of the higher dimensional particle to the many lower dimensional particles in such way that the number of lower dimensional particles becomes

$$
N_{\mathrm{d}-1}=N_{\mathrm{d}} / \alpha^{2} \approx N_{\mathrm{d}}(137)^{2}
$$

The fractionalization also applies to D for 10D4d to 9D4d, so

$$
N_{\text {D-1 }}=N_{\mathrm{D}} / \alpha^{2}
$$

Since the supersymmetry transformation involves translation, this stepwise varying supersymmetry transformation leads to a translational fractionalization, resulting in the cosmic expansion. Afterward, the QVSL transformation transforms 9D4d into 8D5d with a higher mass. The two-step transformation repeats until 4D4d, and then reverses stepwise back to 10D4d for the cosmic contraction. The oscillation between 10D and 4D results in the reversible cyclic fractionalization-contraction for the reversible cyclic expansion-contraction of the universe which does not involve irreversible kinetic energy.

\section{The Digital Transitional Higgs-Reverse Higgs Fields Postulate for the Digital Space Structure}

In the conversion model, under spontaneous symmetry breaking, zero-energy ground state space turns into the nonzero-energy scalar Higgs Field which exists permanently in the universe. The problem with such nonzeroenergy field is the cosmological constant problem from the huge gravitational effect by the nonzero-energy Higgs field [5]. The coupling of massless particle to the Higgs field produces the transitional nonzero-energy Higgs field-particle composite which under spontaneous symmetry restoring produces the massive particle with the longitudinal component on zero-energy ground state space without the Higgs field as follows.

$$
\begin{aligned}
& \stackrel{\substack{\text { zerro-energy groud state space } \stackrel{\text { spontaneous symmetry breaking }}{\longrightarrow} \text { nonzero-energy scalar Higgs field } \\
\text { massless particle }}}{\stackrel{\text { spontaneous symmetry restoring }}{\longrightarrow} \text { the transitional nonzero- energy Higgs field-particle composite] }} \text { massive particle with the longitudinal component on }
\end{aligned}
$$

zero-energy ground state space without the Higgs field

In this paper, to avoid the cosmological problem from the huge gravitational effect by the nonzero-energy Higgs field is to make the Higgs field a transitional field which exists momentarily and to make zero-energy ground state space a permanent zero-energy ground state space which exists permanently in the universe. In the second postulate for the digital structure, such zero-energy ground state space is zero-energy "attachment space" which attaches particles to account for the longitudinal component, mass, and reversible movement. Unlike the conventional model, attachment space actively couples to massless particle. Under spontaneous symmetry breaking, the coupling of massless particle to zero-energy attachment space produces the transitional nonzeroenergy Higgs field-particle composite which under spontaneous symmetry restoring produces massive particle on zero-energy attachment space with the longitudinal component without the Higgs field as follows.

$$
\begin{aligned}
& \text { massless particle + zero-energy attachment space } \stackrel{\text { spontaneous symmetry breaking }}{\longrightarrow} \\
& \text { [the transitional non-zero energy Higgs field-particle composite] } \\
& \stackrel{\text { spontaneoussymmetry restoring }}{\longrightarrow} \text { massive particle with the longitudinal component }
\end{aligned}
$$

on zero-energy attachment space without the Higgs field

The opposite of attachment space is zero-energy detachment space which detaches particles to account for irreversible kinetic energy. Unlike the conventional model, detachment space actively couples to massive particle. Under spontaneous symmetry breaking, the coupling of massive particle to zero-energy detachment space produces the transitional nonzero-energy reverse Higgs field-particle composite which under spontaneous symme- 
try restoring produces massless particle on zero-energy detachment space without the longitudinal component without the reverse Higgs field as follows.

$$
\begin{aligned}
& \text { massive particle + zero-energy detachment space } \stackrel{\text { spontaneoussymmetry breaking }}{\longrightarrow} \\
& \text { [the transitional nonzero-energy reverse Higgs field-particle composite] } \\
& \underset{\text { spontaneoussymmetryrestoring }}{\longrightarrow} \text { massless particle without the longitudinal } \\
& \text { compnenent on zero-energy detachment space without the reverse Higgs field }
\end{aligned}
$$

For the electroweak interaction in the Standard model where the electromagnetic interaction and the weak interaction are combined into one symmetry group, under spontaneous symmetry breaking, the coupling of the massless weak W, weak Z, and electromagnetic A (photon) bosons to zero-energy attachment space produces the transitional nonzero-energy Higgs fields-bosons composites which under partial spontaneous symmetry restoring produce massive $\mathrm{W}$ and $\mathrm{Z}$ bosons on zero-energy attachment space with the longitudinal component without the Higgs field, massless A (photon), and massive Higgs boson as follows.

$$
\begin{aligned}
& \text { massless WZ + zero-energy WZ attachment space + massless A } \\
& \text { +zero-energy A attachment space A } \stackrel{\text { spontaneoussymmetry breaking }}{\longrightarrow} \\
& \text { [the transitional nonzero-energy WZ Higgs field -WZ composite] } \\
& +[\text { nonzero-energy A Higgs field -A composite] }] \stackrel{\text { partialspontaneoussymmetry restoring }}{\longrightarrow} \\
& \text { massive WZ with the longitudinal component on attachment space without } \\
& \text { the Higgs field + massless A + the nonzero energy massive Higgs boson }
\end{aligned}
$$

From the periodic table of elementary particles, the theoretical calculated mass of the Higgs boson is 128.8 $\mathrm{GeV}$ in good agreements with the observed 125 or $126 \mathrm{GeV}$ [3]. In terms of mathematical expression, the conventional permanent Higgs field model and the posited transitional Higgs field model are identical. The interpretations of the mathematical expression are different for the permanent Higgs field model and the transitional Higgs field model. The transitional Higgs field model avoids the cosmological problem in the permanent Higgs field model.

As shown later, our universe is the dual asymmetrical positive-energy-negative-energy universe where the positive-energy universe on attachment space absorbed the interuniversal void on detachment space to result in the combination of attachment space and detachment space, and the negative-energy universe did not absorb the interuniversal void. The combination of $\mathrm{n}$ units of attachment space as 1 and $\mathrm{n}$ units of detachment space as 0 brings about three different digital space structures: binary partition space, miscible space, or binary lattice space as below.

$$
(1)_{n} \quad+\quad(0)_{n} \quad \stackrel{\text { combination }}{\longrightarrow}(1)_{n}(0)_{n}, \quad(1+0)_{n}, \quad \text { or } \quad(10)_{n}
$$

attachment space detachment space binary partition space, miscible space, binary lattice space

Binary partition space, $(1)_{\mathrm{n}}(0)_{\mathrm{n}}$, consists of two separated continuous phases of multiple quantized units of attachment space and detachment space. In miscible space, $(1+0)_{\mathrm{n}}$, attachment space is miscible to detachment space, and there is no separation of attachment space and detachment space. Binary lattice space, $(10)_{n}$, consists of repetitive units of alternative attachment space and detachment space.

Binary partition space is the space for wavefunction in quantum mechanics. In wavefunction,

$$
|\Psi\rangle=\sum_{i=1}^{n} c_{i}\left|\phi_{i}\right\rangle
$$

Each basis element, $\left|\phi_{i}\right\rangle$, has both attachment space and detachment space as binary partition space. Neither attachment space nor detachment space is zero in binary partition space for a basic element. The measurement in the uncertainty principle in quantum mechanics is essentially the measurement of attachment space size and momentum from the detachment space in binary partition space: large momentum from detachment space has small non-zero attachment space size, while large attachment space size has low non-zero momentum from detachment space. In binary partition space, an entity is both in constant motion as wave for detachment space and in stationary state as a particle for attachment space, resulting in the wave-particle duality. 
In binary partition space, for every detachment space, there is its corresponding adjacent attachment space. Thus, no part of the mass-energy can be irreversibly separated from binary partition space, and no part of a different mass-energy can be incorporated in binary partition space. Binary partition space represents coherence as wave function. Binary partition space is for coherent system. Any destruction of the coherence by the addition of a different mass-energy to the mass-energy causes the collapse of binary partition space into miscible space. The collapse is a phase transition from binary partition space to miscible space.

$$
\begin{array}{ll}
\stackrel{(0)_{n}(1)_{n} \stackrel{\text { collapse }}{\longrightarrow}}{\longrightarrow}(0+1)_{n} \\
\text { binary partition space } \quad \text { miscible space }
\end{array}
$$

The information in miscible space is contributed by the miscible combination of both attachment space and detachment space, so information can no longer be non-localized. Any value in miscible space is definite. All observations in terms of measurements bring about the collapse of wavefunction, resulting in miscible space that leads to eigenvalue as definite quantized value. Such collapse corresponds to the appearance of eigenvalue, E, by a measurement operator, $H$, on a wavefunction, $\Psi$.

$$
H \Psi=E \Psi
$$

In miscible space, attachment space is miscible to detachment space, and there is no separation of attachment space and detachment space. In miscible space, attachment space contributes zero speed, while detachment space contributes the speed of light. For a moving massive particle consisting of a rest massive part and a massless part, the massive part with rest mass, $m_{0}$, is in attachment space, and the massless part with kinetic energy, $K$, is adjacent to detachment space. The combination of the massive part in attachment space and massless part in detachment leads to the propagation speed in between zero and the speed of light. To maintain the speed of light constant for a moving particle, the time $(t)$ in moving particle has to be dilated, and the length $(L)$ has to be contracted relative to the rest frame.

$$
\begin{aligned}
& t=t_{0} / \sqrt{1-v^{2} / c^{2}}=t_{0} \gamma, \\
& L=L_{0} / \gamma, \\
& E=K+m_{0} c^{2}=\gamma m_{0} c^{2}
\end{aligned}
$$

where $\gamma=1 /\left(1-v^{2} / c^{2}\right)^{1 / 2}$ is the Lorentz factor for time dilation, and length contraction, $E$ is the total energy, and $K$ is the kinetic energy.

Bounias and Krasnoholovets [18] propose that the reduction of dimension can be done by slicing dimension, such as slicing 3 space dimension object (block) into infinite units of 2 space dimension objects (sheets). As shown in Section 4, the positive-energy 10D4d particle universe as our observable universe with high vacuum energy was transformed into the 4D10d universe with zero vacuum energy at once, resulting in the inflation. During the Big Bang following the inflation, the 10d (mass dimension) particle in attachment space denoted as 1 was sliced by detachment space denoted as 0 . For example, the slicing of $10 \mathrm{~d}$ particle into $4 \mathrm{~d}$ particle is as follows.

$$
\begin{array}{cll}
1_{10} \stackrel{\text { slicing }}{\longrightarrow} 1_{4} \quad \sum_{\mathrm{d}=5}^{10}\left(0_{4} 1_{4}\right)_{n, \mathrm{~d}} \\
\text { 10d particle } & \text { 4d core particle } & \text { binary lattice space }
\end{array}
$$

where $1_{10}$ is $10 \mathrm{~d}$ particle, $1_{4}$ is $4 \mathrm{~d}$ particle, $\mathrm{d}$ is the mass dimension number of the dimension to be sliced, $\mathrm{n}$ as the number of slices for each dimension, and $\left(0_{4} 1_{4}\right)_{\mathrm{n}}$ is binary lattice space with repetitive units of alternative $4 \mathrm{~d}$ attachment space and $4 \mathrm{~d}$ detachment space. For $4 \mathrm{~d}$ particle starting from $10 \mathrm{~d}$ particle, the mass dimension number of the dimension to be sliced is from $d=5$ to $d=10$. Each mass dimension is sliced into infinite quantized units $(n=$ $\infty$ ) of binary lattice space, $\left(0_{4} 1_{4}\right)_{\infty}$. For $4 d$ particle, the $4 d$ core particle is surrounded by 6 types (from $d=5$ to $d=$ 10) of infinite quantized units of binary lattice space. Such infinite quantized units of binary lattice space represent the infinite units $(n=\infty)$ of separate virtual orbitals in a gauge force field, while the dimension to be sliced is "dimensional orbital” (DO), representing a type of gauge force field. The mass-energy in each dimensional orbital increases with the number of dimension number, and the lowest dimension orbital with $d=5$ has the lowest mass-energy [2] [11]. 10d particle was sliced into six different particles: 9d, 8d, 7d, 6d, 5d, and 4d equally by mass. Baryonic matter is $4 d$, while dark matter consists of the other five types of particles (9d, 8d, 7d, 6d, and 5d). 


$$
\begin{aligned}
& \text { 10D4d } \stackrel{\text { the inflation }}{\longrightarrow} \text { 4D10d } \stackrel{\text { the Big Bang }}{\longrightarrow} \text { baryonic matter (4D4d) } \\
& + \text { dark matter (4D5d, 4D6d, 4D7d, 4D8d, 4D9d) + kinetic energy }
\end{aligned}
$$

The mass ratio of dark matter to baryonic matter is 5 to 1 . At $72.8 \%$ dark energy, the calculated values for baryonic matter and dark matter (with the $1: 5$ ratio) are $4.53 \%(=(100-72.8) / 6)$ and $22.7 \%(=4.53 \times 5)$, respectively, in excellent agreement with observed $4.56 \%$ and $22.7 \%$, respectively [11] [19]. The dimensional orbitals of baryonic matter provide the base for the periodic table of elementary particles to calculate accurately the masses of all elementary particles, including quarks, leptons, gauge bosons, the Higgs boson, and the kneesankles-toe in cosmic rays [2]-[4]. The calculated masses of all elementary particles are in good agreement with the observed values. For examples, the calculated mass of top quark and the Higgs boson are $176.5 \mathrm{GeV}$ and $128.8 \mathrm{GeV}$ in good agreement with the observed $173.34 \mathrm{GeV}$ and 125 or $126 \mathrm{GeV}$, respectively.

The lowest dimensional orbital is for electromagnetism. Baryonic matter with maximum number of gauge force fields (dimensional orbitals) is the only one with the lowest dimensional orbital for electromagnetism. With higher dimensional orbitals, dark matter does not have this lowest dimensional orbital [6] [12]. Without electromagnetism, dark matter cannot emit light, and is incompatible to baryonic matter with electromagnetism, like the incompatibility between oil and water. Derived from the incompatibility between dark matter and baryonic matter, the modified interfacial gravity (MIG) between homogeneous baryonic matter region and homogeneous dark matter region to separate baryonic matter region and dark matter region explains galaxy evolution and unifies the CDM (Cold Dark Matter) model, MOG (Modified Gravity), and MOND (Modified Newtonian Dynamics) [13] [14]. The digital space structure based on the combination of binary partition space and binary lattice space explains superconductivity [15] and superstar without singularity to replace black hole with singularity [16] [17]. Singularity is permanently irreversible by losing information permanently, forbidden in the reversible multiverse.

\section{Cosmology and the Reversible Multiverse Postulate for All Physical Laws and Phenomena}

In the reversible multiverse postulate, all physical laws and phenomena are permanently reversible, and temporary irreversibility of entropy increase is allowed through reversibility breaking, symmetry violation, and low entropy beginning. The reversible multiverse postulate can be explained by cosmology. The multiverse has been studied extensively. For example, Brian Greene [20] described the nine types of the multiverse which produce complicated collections of universes. The reversible multiverse postulate posits a simple version of the multiverse. The posited simple multiverse is the reversible multiverse that excludes any permanently irreversible physical laws and phenomena. In the reversible multiverse, the allowed universes have to be reversible cyclic universes with permanently reversible physical laws and phenomena, resulting in only limited types of allowed universes. Temporary irreversible universes are allowed. One irreversible phenomenon which is not allowed is the collision of expanding universes. The collision of expanding universes which have the inexhaustible resource of space-time to expand is permanently irreversible due to the impossibility to reverse the collision of expanding universes. To prevent the collision of expanding universes, every universe is surrounded by the interuniversal void that is functioned as the permanent gap among universes. The interuniversal void has zeroenergy, zero space-time, and zero vacuum energy, and detachment space only, while universe has nonzeroenergy, the inexhaustible resource of space-time to expand, zero or/and non-zero vacuum energy, and attachment space with or without detachment space. The detachment space of the interuniversal void has no spacetime, so it cannot couple to particles with space-time in universes, but it prevents the advance of expanding universes into the interuniversal void to avoid the collision of expanding universes.

A zero-sum energy dual universe of positive-energy universe and negative-energy universe can be created in the zero-energy interuniversal void, and the new dual universe is again surrounded by the interuniversal void to avoid the collision of universes. Under symmetry, the new positive-energy universe and the new negativeenergy universe undergo mutual annihilation to reverse to the interuniversal void immediately. Our universe is the dual asymmetrical positive-energy-negative-energy universe where the positive-energy universe on attachment space absorbed the interuniversal void on detachment space to result in the combination of attachment space and detachment space, and the negative-energy universe did not absorb the interuniversal void. Within the positive-energy universe, the absorbed detachment space with space-time can couple to particles in the positive- 
energy universe to result in massless particles with irreversible kinetic energy. The formation of our universe involves symmetry violation between the positive-energy universe and the negative energy universe. Irreversible kinetic energy from detachment space is the source of irreversible entropy increase, so the positive-energy universe is locally irreversible, while the negative-energy universe without irreversible kinetic energy from detachment space is locally reversible. The locally reversible negative-energy universe guides the reversible process of the dual universe. As a result, our whole dual universe is globally reversible. Our dual universe is the globally reversible cyclic dual universe as shown in Equation (16) and Figure 1 for the evolution of our universe.

$$
\begin{aligned}
& \text { the dual positive energy - negative energy universe } \stackrel{\text { symmetry breaking-reversibility breaking }}{\longrightarrow} \\
& \text { the locally irreversible positive energy universe }+ \text { the locally reversible negative energy universe } \\
& \rightarrow \text { the globally reversible cyclic dual universe }
\end{aligned}
$$

The four reversible steps in the globally reversible cyclic dual universe are 1) the formation of the 11D membrane dual universe, 2) the formation of the 10D string dual universe, 3) the formation of the 10D particle dual universe, and 4) the formation of the asymmetrical dual universe.

1) The formation of the 11D membrane dual universe

As described previously [7] [9]-[11], the reversible cyclic universe starts in the zero-energy interuniversal void, which produces the dual universe of the positive-energy 11D membrane universe and the negative-energy 11D membrane universe as in Figure 1. In some dual 11D membrane universes, the 11D positive-energy membrane universe and the negative-energy 11D membrane universe coalesce to undergo annihilation and to return to the interuniversal void as in Figure 1.

2) The formation of the 10D string dual universe

Under the reversible oscillation between 11D and 10D, the positive-energy11D membrane universe and the negative-energy 11D membrane universe are transformed into the positive-energy 10D string universe and the negative-energy 10D string universe, respectively, as in Figure 1. The positive-energy 11D membrane universe is transformed into the positive-energy 10D string universe as in Equations (17a) and (17b).

$$
\begin{aligned}
& \text { The RS1Membrane Transformation } \\
& \text { step } 1: 2_{11} \stackrel{\text { from 11D membrane to 10D string }}{\longrightarrow} 1_{10} \text { in the11D AdS space } \\
& \text { step } 2: 2\left(1_{10}\right) \stackrel{\text { the closestring vibration }}{\longrightarrow} 1_{10} 0_{10}=1_{10} g_{e} \text { in the11D AdS space } \\
& 2\left(2_{11}\right) \stackrel{\text { the closestring and theopen string vibrations }}{\longrightarrow}\left(s 1_{10}\right) g_{e}
\end{aligned}
$$

where $2_{11}$ is membrane (denoted as 2) in $11 \mathrm{D}, s$ is the pre-strong force, $1_{10}$ is string (denoted as 1 ) in $10 \mathrm{D}, 0_{10}$ is particle (denoted as 0 ) in 10D, AdS is anti-de Sitter, and $g_{e}$ is the external graviton.

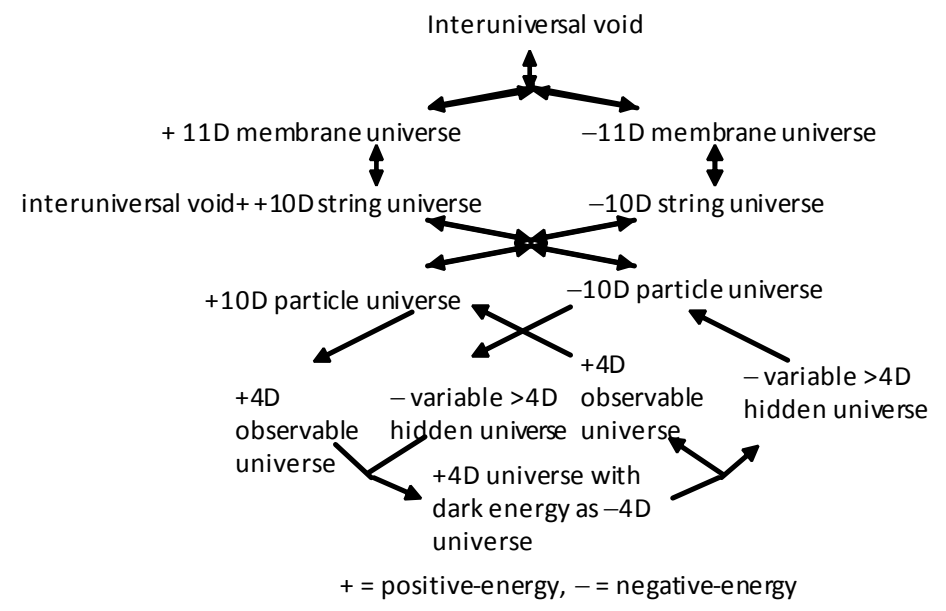

Figure 1. The globally reversible cyclic dual universe. 
According Randall and Sundrum, the RS1 (Randall-Sundrum model 1) in an anti-de Sitter (AdS) space consists of one brane with extremely low graviton's probability function and another brane with extreme high graviton's probability function [21] [22]. The formation of the 10D string dual universe involves the RS1. As shown in Equation (17a), one of the possible membrane transformations from the 11D membrane to the 10D string is the RS1 membrane transformation which involves two steps. In the Step 1, the extra spatial dimension of the 11D membrane in the transformation from the 11D membrane to the 10D string becomes the spatial dimension transverse to the string brane in the bulk 11D anti-de Sitter space [21]. This transformation is derived from the transformation from membrane to string. In the transformation from the two-dimensional membrane to the one-dimensional string, the extra spatial dimension of the two-dimensional membrane on the $x-y$ plane becomes the $\mathrm{x}$-axis transverse to the one-dimensional string on the $\mathrm{y}$-axis in the two-dimensional $\mathrm{x}$-y space. In the Step 2, for the RS1 membrane transformation, two string branes are combined into the combined string brane. The external 10D particles generated by the close string vibration of the combined string brane are the 10D external gravitons which form the external graviton brane as the Gravity brane (Planck Plane) in the RS1 of the Randall-Sundrum model [21] [22]. As in the RS1 of the Randall-Sundrum model, the two branes with equal mass-energy in the 11D anti-de Sitter space are the string brane with weak gravity and the external graviton brane with strong gravity. The weak gravity in the string brane is the predecessor of the observed weak gravity generated during the Big Bang [2] [9]. The external graviton in the external graviton brane is the predecessor of a part of the observed dark energy [11]. The 10D string brane and the 10D external graviton brane correspond to the predecessors of the observed universe (without dark energy) and a part of observed dark energy, respectively [2] [9]. The reverse transformation from 10D to 11D is the RS1 string transformation.

In Equation (17b), the particles generated from the 10D open string vibration are the 10D particles for the prestrong force (denoted as $s$ ) in addition to the external graviton from the close string vibration in the 11D AdS. According to Maldacena, the AdS/CFT correspondence is a correspondence between quantum gravity in AdS space and quantum field theory of conformal field theory (CFT) in one dimension lower [23]. The AdS/CFT correspondence describes Equation (17b) as the correspondence between the external graviton in the 11D AdS and the pre-strong force of 10D CFTin one dimension lower. The pre-strong force is the same for all strings without positive or negative sign. This pre-strong force is the prototype of the observed strong force generated during the Big Bang [2] [9].

In the negative universe through symmetry, the $11 \mathrm{D}$ anti-membrane $\left(2_{-11}\right)$ is transformed into10D antistring $\left(1_{-10}\right)$ with external anti-graviton $g_{e}$ and the pre-strong force $\mathrm{s}$ as follows.

$$
2\left(2_{-11}\right) \leftrightarrow\left(s 1_{-10}\right) \overline{g_{e}}
$$

The dual universe of the positive-energy 10D string universe with $n$ units of $\left(1_{10}\right)_{n}$ and the negative-energy 10D string universe with $\mathrm{n}$ units of $\left(1_{-10}\right)_{n}$ is as follows.

$$
\left(\left(s 1_{10}\right) g_{e}\right)_{n}\left(\overline{g_{e}}\left(s 1_{-10}\right)\right)_{n}
$$

There are four equal regions: the positive-energy 10D string universe, the external graviton, the external antigraviton, and the negative-energy $10 \mathrm{D}$ string universe.

Some dual 10D string universes return to the dual 11D membrane universes under the reversible oscillation between 11D and 10D. Alternatively, under symmetry violation as in the case of our universe, the positive-energy $10 \mathrm{D}$ string universe absorbs the interuniversal void, while the negative-energy $10 \mathrm{D}$ string universe does not absorb the interuniversal void. The interuniversal void has zero vacuum energy. In our universe, the absorption of the interuniversal void by the positive-energy 10D string universe forced the positive-energy 10D universe with high vacuum energy to be transformed into the universe with zero vacuum energy that was the vacuum energy of the 4D universe. However, the transformation from 10D to 4D was not immediate, because the strings had to be 10D, and it could not be transformed into 4D, therefore, strings had to be transformed into particles that allowed the change of its dimension number freely to accommodate the transformation from the 10D universe to the 4D universe driven by the absorption of the interuniversal void.

3) The formation of the 10D particle dual universe

As described previously [9], the transformation of strings into particles came from the emergence of positive charge and negative charge that allowed the mutual annihilation of positively charged 10D strings and negatively charged 10D antistrings in the 10D string universes to produce positively charged 10D particles and negatively 
pre-charged 10D antiparticles in the 10D particle universes as follows.

$$
\left(\left(s 0_{10} e^{+} e^{-} 0_{-10} s\right) g_{e}\right)_{n}\left(\overline{g_{e}}\left(s 0_{10} e^{+} e^{-} 0_{-10} s\right)\right)_{n},
$$

where $s$ and $e$ are the pre-strong force and the pre-charged force in the flat space, $g_{e}$ is the external graviton, $g_{e}$ is the external graviton, and $0_{10} 0_{-10}$ is the particle-antiparticle. There are four equal regions: the $10 \mathrm{D}$ positive-energy particle universe, the external graviton, the 10D negative-energy particle universe, and the external anti-graviton. The emergence of positive charge and negative charge provides the prototype of the observed electromagnetic force with charge generated during the Big Bang [2] [9].

4) The formation of the asymmetrical dual universe

The formation of our current universe follows immediately after the formation of the 10D particle dual universe through the asymmetrical dimensional oscillations, leading to the asymmetrical dual universe. The 10D positive-energy universe was transformed immediately into the 4D positive-energy particle universe with zero vacuum energy. The 10D negative-energy particle universe undergoes the stepwise dimension number oscillation between 10D and 4D. Without absorbing the interuniversal void, the external graviton and the anti-graviton also undergo the stepwise dimension number oscillation between 10D and 4D. The result is the asymmetrical dual universe consisting of the four equal regions of the $4 \mathrm{D}$ positive-energy particle universe, the variable $\mathrm{D}$ external graviton, the variable $\mathrm{D}$ negative-energy particle universe, and the variable $\mathrm{D}$ external anti-graviton. The asymmetrical dual universe is manifested as the asymmetry in the weak interaction in our observable universe as follows.

the $4 \mathrm{D}$ positive-energy particle universe and the external graviton

$$
\left(\left(s 0_{4} e^{+} w^{+} e^{-} w^{-} 0_{-4} s\right) g_{e}\right)_{n}
$$

the variable $D$ negative-energy particle universe and the external anti-graviton

$$
\left(\overline{g_{e}}\left(s 0_{4 \text { to10 }} e^{+} w^{+} e^{-} w^{-} 0_{-4 t 0-10} s\right)\right)_{n}
$$

where $s, g_{e}, g_{e}, e$, and $w$ are the strong force, external graviton, external anti-graviton, electromagnetism, and weak interaction, respectively for the observable universe, and where $0_{4} 0_{-4}$ and $0_{4}$ to $100_{-4}$ to -10 are $4 \mathrm{D}$ particleantiparticle for the $4 \mathrm{D}$ positive-energy particle universe and variable $\mathrm{D}$ particle-antiparticle for the variable $\mathrm{D}$ negative-energy particle universe, respectively. For our asymmetrical dual universe, the step 3 for the transformation of 10D string into 10D particle had to be followed by the step 4, so the electromagnetic interaction from the step 3 was unified with the weak interaction from the step 4 to become the electroweak interaction, which was generated during the Big Bang [2] [9].

a) The formation of the 4D positive-energy particle universe

The formation of 4D positive-energy particle universe involved the two-step transformation: 1.the inflation and 2 , the Big Bang. In the first step, the inflation is the transformation from 10D4d to 4D10d immediately. Calculated from Equation (1e), the rest mass of $4 \mathrm{D} 10 \mathrm{~d}$ is $1 / \alpha^{12} \approx 137^{12}$ times of the mass of $10 \mathrm{D} 4 \mathrm{~d}$, resulting in the first step of the inflation as the rapid expansion of space from the high vacuum energy 10D4d to the zero vacuum energy 4D10d as follows.

\section{1. the inflation}

$$
\text { 10D4d } \stackrel{\text { quick QVSL transformation }}{\longrightarrow} 4 \mathrm{D} 10 \mathrm{~d}
$$

In the second step of the transformation, the Big Bang is a two-step process. The first step is the coupling of detachment space and the massive particles on attachment space in the positive-energy universe that absorbed the interuniversal void on detachment space. The result is the total conversion to generate massless particles on detachment space in the positive-energy universe and the external attachment space surrounded the positiveenergy universe as described in Equation (7). In the second step, the coupling of attachment space and the massless particles in the positive-energy universe that absorbed the external attachment space surrounded the positive-energy universe. The partial conversion resulted in massive particles such as weak bosons, leptons, the Higgs boson, and massless particles such as photon. The second step is described in Equation (8) through the Higgs mechanism. The irreversible kinetic energy resulted from detachment space started the positive-energy universal expansion. The positive-energy universe has the combination of attachment space and detachment as follows. 


\section{The Big Bang}

1. massive particles on attachment space + detachment space $\stackrel{\text { total conversion }}{\longrightarrow}$ massless particles on detachment space + the external attachment space

2. massless particles on detachment space +the external attachment space $\stackrel{\text { partial conversion }}{\longrightarrow}$

massless particles + massive particles + detachment space + attachment space + the Higgs boson

The presence of irreversible kinetic energy induces irreversible entropy increase. In the Boltzmann formula of thermodynamic, the absolute entropy $S$ of an ideal gas to the quantity $W$, which is the number of the arrangements of particles corresponding to a given macroscopic collection of particles:

$$
S=k_{B} \ln W
$$

where $k_{B}$ is the Boltzmann's constant. The Boltzmann formula shows the relationship between entropy and the number of ways the atoms or molecules of a thermodynamic system can be arranged. The various atoms or molecules have different positions and momenta for irreversible entropy increase, because the increase in the number of different arrangements of particles in a macroscopic collection of particles requires the movements of individual particles in a macroscopic collection of particles. (There is no entropy increase in a single microscopic particle.) Individual momenta from kinetic energy are required for irreversible entropy increase in a macroscopic collection of particles. In other words, kinetic energy transforms a macroscopic collection of particles from one way of the arrangement of particles (order) into many ways of the arrangements of particles (disorder), and the process from order to disorder is irreversible in an isolated macroscopic collection of moving particles.

In our universe, the interuniversal void on detachment space was by the $10 \mathrm{D}$ positive-energy string universe which was very small. (The rest mass of $4 \mathrm{D} 10 \mathrm{~d}$ is $1 / \alpha^{12} \approx 137^{12}$ times of the mass of $10 \mathrm{D} 4 \mathrm{~d}$.) To have exactly reversible absorption-desorption of the interuniversal void for the reversible dual universe, the absorption and desorption have to be uniform. The space of the universe where the absorption-desorption occurs has to be small enough for the uniform absorption-desorption. The 10D string was small mass to allow the uniform absorptiondesorption for reversible absorption-desorption of the interuniversal void, resulting in low entropy beginning. (The subsequent irreversible absorption of the interuniversal void is forbidden.) In the reversible multiverse postulate, all physical laws and phenomena are permanently reversible, and temporary irreversibility of entropy increase is allowed through reversibility breaking, symmetry violation, and low entropy beginning. Our 4D positive-energy particle universe is an example of irreversibility of entropy increase through reversibility breaking, symmetry violation, and low entropy beginning.

b) The formation of the variable D negative-energy particle universe

The formation of the variable D negative-energy particle universe involves the stepwise two-step transformation: the QVSL transformation and the varying supersymmetry transformation from 10D4d to 4D4d. (The particles in the 10D dual particle universe are 10D4d.) The QVSL transformation involves the transformation of space-time dimension, D. The repetitive stepwise two-step transformation from 10D4d to 4D10d as follows.

$$
\begin{aligned}
& \text { 10D4d } \rightarrow \text { 9D5d } \rightarrow \text { 9D4d } \rightarrow \text { 8D5d } \rightarrow \cdots \rightarrow \text { 4D5d } \rightarrow \text { 4D4d } \\
& \mapsto \text { hidden dark } \quad \text { universe } \leftarrow \mapsto \text { dark energy } \leftarrow
\end{aligned}
$$

The variable D negative-energy particle universe consists of two periods: the hidden variable D negative-energy particle universe and the dark energy universe. The hidden variable $\mathrm{D}$ negative-energy particle universe composes of the $>4 \mathrm{D}$ particles. As mentioned before, particles with different space-time dimensions are transparent and oblivious to one another, and separate from one another if possible. Thus, $>4 \mathrm{D}$ particles are hidden and separated particles with respect to $4 \mathrm{D}$ particles in the $4 \mathrm{D}$ positive-energy particle universe (our observable universe). The hidden variable $\mathrm{D}$ negative-energy particle universe with $\mathrm{D}>4$ and the observable universe with $\mathrm{D}=4$ are the "parallel universes". The 4D particles transformed from hidden $>4 \mathrm{D}$ particles in the variable D negative-energy particle universe are observable dark energy for the 4D positive-energy particle universe, resulting in the accelerated expanding universe. Since the variable D negative-energy particle universe does not have detachment space, the presence of dark energy is not different from the presence of the cosmological constant. According to the theoretical calculation based on the asymmetrical dual universe, dark energy started in 4.47 billion years ago in agreement with the observed $4.71 \pm 0.98$ billion years ago [11]. Our asymmetrical dual universe consists of the four equal regions of the $4 \mathrm{D}$ positive-energy particle universe, the variable $\mathrm{D}$ external graviton, the variable $\mathrm{D}$ 
negative-energy particle universe, and the variable D external anti-graviton, so the percentage the variable D area is $75 \%$, three out of four regions, as the maximum percentage of dark energy. In terms of quintessence, such dark energy can be considered the tracking quintessence [23] [24] from the variable D area with the space-time dimension number as the tracker.

After the maximally connected universe, 4D dark energy transforms back to $>4 \mathrm{D}$ particles that are not observable. The removal of dark energy in the observable universe results in the stop of accelerated expansion and the start of contraction of the observable universe. The end of dark energy starts another "parallel universe period". Both hidden universe and observable universe contract synchronically and equally. Eventually, the Big Crush and the two-step deflation occur in the 4D positive-energy particle universe. In the first step of the deflation, the 4D positive-energy particle universe loses all detachment space, kinetic energy, light, cosmic radiation, and force fields as dimensional orbitals, resulting in returning to 4D10d. In the second step of the deflation, without irreversible kinetic energy, the reversible direct dimension number oscillation resumes to transform the low vacuum energy 4D10d into the high vacuum energy 10D4d for the rapid contraction of space. Meanwhile, hidden $>4 \mathrm{D}$ particles-antiparticles in the hidden universe transform into 10D4d particles-antiparticles. The dual universe can undergo another cycle of the light-dark dual universe. On the other hand, both universes can undergo the reverse charge transformation to become the 10D dual string universe, which in turn can return to the 11D dual membrane universe that in turn can return to the zero-energy universe as Figure 1.

\section{Summary}

The posited theory of everything is the dynamic and reversible process of continuingly changing matters and spaces in the reversible multiverse. Instead of remaining fixed, space-time dimension numbers of matters oscillate. Instead of being passive, spaces actively couple to particles. There are two different spaces whose digital combinations are different under different conditions. In the midst of continuously changing matters and spaces, the multiverse is simple and neat, because the multiverse is permanently reversible to exclude all irreversible physical laws and phenomena. However, temporary irreversible entropy increase is allowed through reversibility breaking, symmetry violation, and low entropy beginning. We live in the universe with such temporary irreversible entropy increase.

The dynamic and reversible theory of everything consists of the three postulates as 1) the oscillating M-theory postulate for the oscillating matter structure, 2) the digital transitional Higgs-reversed Higgs fields postulate for the digital space structure, and 3) the reversible multiverse postulate for all physical laws and phenomena. Oscillating M-theory is derived from oscillating membrane-string-particle whose space-time dimension number oscillates between 11D and 10D and between 10D and 4D. Space-time dimension number between 10 and 4 decreases with decreasing speed of light, decreasing vacuum energy, and increasing rest mass. The digital transitional Higgs-reversed Higgs fields are derived from digital attachment-detachment spaces which couple to particles. Under spontaneous symmetry breaking, the coupling of massless particle to zero-energy attachment space (the space for mass) produces the transitional nonzero-energy Higgs field-particle composite which under spontaneous symmetry restoring produces massive particle on zero-energy attachment space with the longitudinal component. The opposite of attachment space is detachment space as the space for kinetic energy and the nonzero-energy reverse Higgs field. The combination of $n$ units of attachment space (denoted as 1 ) and $n$ units of detachment space (denoted as 0 ) brings about the three digital structures: binary partition space $(1)_{n}(0)_{n}$, miscible space $(1+0)_{n}$, and binary lattice space $\left(\begin{array}{ll}1 & 0\end{array}\right)_{\mathrm{n}}$ to account for quantum mechanics, special relativity, and the force fields, respectively. In the third postulate, all physical laws and phenomena are permanently reversible in the multiverse, and temporary irreversible entropy increase is allowed.

Our universe is an asymmetrical dual positive-energy-negative-energy universe where the positive-energy universe on attachment space absorbed the interuniversal void on detachment space to result in the combination of attachment space and detachment space, while the negative-energy universe did not absorb the interuniversal void, resulting in temporary irreversible entropy increase through reversibility breaking, symmetry violation, and low entropy beginning. Guided by the reversible negative-energy universe, our dual universe is a globally reversible cyclic dual universe. The four reversible steps in the globally reversible cyclic dual universe are 1) the formation of the 11D membrane dual universe, 2) the formation of the 10D string dual universe, 3) the formation of the 10D particle dual universe, and 4) the formation of the asymmetrical dual universe. The four force fields (gravity, the strong force, electromagnetism, and the weak force) are derived from the 4-step evolution of the cyclic dual un- 
iverse. Baryonic matter and dark matter are in the positive-energy universe, while a part of dark energy is in the negative-energy universe.

The posited theory of everything based on the three postulates explains cosmology, the composition (baryonic matter, dark matter, and dark energy) in the universe, the periodic table of elementary particles (quarks, leptons, and bosons), the galaxy evolution, superconductivity, black hole, thermodynamic, and quantum mechanics.

\section{References}

[1] Woit, P. (2006) Not Even Wrong: The Failure of String Theory and the Search for Unity in Physical Law. Basic Books, New York.

[2] Chung, D. (2014) Journal of Modern Physics, 5, 1234-1243. http://dx.doi.org/10.4236/jmp.2014.514123

[3] Chung, D. and Hefferlinm, R. (2013) Journal of Modern Physics, 4, 21-26. http://dx.doi.org/10.4236/jmp.2013.44A004

[4] Chung, D. (2014) Journal of Modern Physics, 5, 1467-1472. http://dx.doi.org/10.4236/jmp.2014.515148

[5] Steven Weinberg, S. (1989) Review Modern Physics, 61, 1-23. http://dx.doi.org/10.1103/RevModPhys.61.1

[6] Chung, D. and Krasnoholovets, V. (2013) Journal of Modern Physics, 4, 27-31. http://dx.doi.org/10.4236/jmp.2013.44A005

[7] Chung, D. (2015) Journal of Modern Physics, 6, 1820-1832. http://dx.doi.org/10.4236/jmp.2015.613186

[8] Carroll, S. (2010) From Eternity to Here: The Quest for the Ultimate Theory of Time. Dutton, New York.

[9] Chung, D. (2015) Journal of Modern Physics, 6, 1249-1260. http://dx.doi.org/10.4236/jmp.2015.69130

[10] Chung, D. (2015) Journal of Modern Physics, 6, 1189-1194. http://dx.doi.org/10.4236/jmp.2015.69123

[11] Chung, D. and Krasnoholovets, V. (2013) Journal of Modern Physics, 4, 77-84. http://dx.doi.org/10.4236/jmp.2013.47A1009

[12] Chung, D. (2014) Journal of Modern Physics, 5, 464-472. http://dx.doi.org/10.4236/jmp.2014.56056

[13] Chung, D. (2014) International Journal of Astronomy and Astrophysics, 4, 374-383. http://dx.doi.org/10.4236/ijaa.2014.42032

[14] Chung, D. (2015) Global Journal of Science Frontier Research A, 15, 119-125.

[15] Chung, D. (2015) Journal of Modern Physics, 6, 26-36. http://dx.doi.org/10.4236/jmp.2015.61005

[16] Chung, D. and Krasnoholovets, V. (2013) Journal of Modern Physics, 4, 1-6. http://dx.doi.org/10.4236/jmp.2013.47A1001

[17] Chung, D. (2014) Global Journal of Science Frontier Research A, 14, 1-8.

[18] Bounias, M. and Krasnoholovets, V. (2003) The International Journal of Systems and Cybernetics, 32, 1005-1020.

[19] Jarosik, N., Bennett, C.L., Dunkley, J., Gold, B., Greason, M.R., Halpern, M., et al. (2011) The Astrophysical Journal: Supplement Series, 192, 14. http://dx.doi.org/10.1088/0067-0049/192/2/14

[20] Greene, B. (2011) The Hidden Reality: Parallel Universes and the Deep Laws of the Cosmos. Alfred A. Knopf, New York.

[21] Randall, L. (2005) Warped Passages: Unraveling the Mysteries of the Universe's Hidden Dimensions. Harper Collins, New York.

[22] Randall, L. and Sundrum, R. (1999) Physical Review Letters, 83, 3370-3373. http://dx.doi.org/10.1103/PhysRevLett.83.3370

[23] Maldacena, J. (1998) Advances in Theoretical and Mathematical Physics, 2, 231-252. http://dx.doi.org/10.4310/ATMP.1998.v2.n2.a1

[24] Padmanabhan, T. (2003) Physics Reports, 380, 235-320. http://dx.doi.org/10.1016/S0370-1573(03)00120-0 